\title{
ESTUDIO DESCRIPTIVO DE LA FRECUENCIA Y DURACIÓN DE LA PUBLICIDAD ALIMENTARIA EMITIDA EN LA PROGRAMACIÓN DE CANALES DE TELEVISIÓN ASOCIADOS A ANATEL
}

\author{
DESCRIPTIVE STUDY OF THE FREQUENCY AND DURATION \\ OF FOOD ADVERTISEMENT IN THE PROGRAMMING OF THE \\ TELEVISION CHANNELS MEMBERS OF THE ANATEL GROUP
}

\author{
Mirta Crovetto M., Marion Durán T., Mariel Guzmán R., Carla Miranda H. \\ Nutrición y Dietética, Facultad de Ciencias de la Salud. \\ Universidad de Playa Ancha. Valparaíso, Chile.
}

\begin{abstract}
The objective of this study was to analyze television advertisements from different TV channels which are part of the Chilean National Television Association. During one week in January and one week in February of 2010 the advertisements were screened. The analysis selected and classified food's advertising as healthy, moderately healthy and unhealthy based on food's critic nutritional components: total fat, saturated fat, carbohydrates and sodium using as reference a classification chart called "Traffic Light System" and created by the UK Food Standard Agency. Sixty thousand four hundred and twenty five (6.425) advertisements were registered and analyzed accounting for 56 hours and 36 minutes in total. One thousand forty five (1.045) advertisements were identified as food advertisements (16\%) accounting for 7 hours and 42 minutes (14\%) of the total. Nutritional labels of the products were studied in order to classify and select them as described above. From the total of food advertisements it was observed that $64 \%$ were related to unhealthy food; $27 \%$ to moderately healthy and $9 \%$ to healthy food; accounting for respectively $62 \%, 30 \%$ and $8 \%$, respectively, of the total time reported on food advertisement. Food advertisements are mostly unhealthy, promoting food consumption with high levels of fat, sugar and sodium. The broadcasting of this type of advertisements by television promotes the population to follow unhealthy life styles which result in a deterioration of their health.
\end{abstract}

Key words: food advertisement, nutritional labels, fat, sugar and sodium.

Este trabajo fue recibido el 17 de Mayo de 2011 y aceptado para ser publicado el 1 de Julio de 2011.

\section{INTRODUCCIÓN}

En las últimas décadas, Chile ha experimentado una notoria transición epidemiológica, demográfica y nutricional, con un aumento de las expectativas de vida de los chilenos pero a la vez un incremento de las Enfermedades Crónicas No Transmisibles (ECNT) (1 -3).

La creciente prevalencia de obesidad y sobrepeso constituyen una crisis global que amenaza la salud de millones de personas $(4,5)$; datos de la Junta Nacional de Auxilio Escolar y Becas (JUNAEB), en Chile, indican que la obesidad presenta una tendencia creciente en los últimos años, afectando en el 2010, a un $23 \%$ de la población infantil que ingresa a primer año básico (6). Entre los factores que explican esta tendencia al alza, se ha detectado una mala alimentación, una menor actividad física, el gran desarrollo de las estrategias de marketing de la industria alimentaria y empresas de alimentos, y las horas que los niños dedican a ver la televisión (7 - 9).

La televisión, además de producir sedentarismo, interfiere en el tipo de alimentación que tienen los niños y adultos, debido a la forma en que la publicidad persuade e influye en la audiencia $(10,11)$, especialmente, en las opiniones de los niños frente a los productos publicita- 
dos que los convierten en verdaderos consumidores y les crea malos hábitos alimentarios $(12,13)$, dado que la mayoría de los alimentos que se publicitan son de alta densidad energética, ricos en grasas saturadas, azúcares agregados y sal, incentivando su consumo y contribuyendo a la obesidad. La población infantil es uno de los grupos objetivos de la publicidad de alimentos de la TV, son clientes, compradores y consumidores, constituyen un grupo fácil de persuadir y son más vulnerables que los adultos en su exposición a la publicidad televisiva, siendo incapaces de comprender que el propósito de un anuncio no es informar sino persuadir, para lograr la venta del producto publicitado (14 - 16). Consumers International (CI) indica que la mayoría de los niños menores de 5 años tienen dificultad para distinguir entre lo que es un anuncio y lo que es un programa y antes de los 8 años no entienden que la intención de un anuncio es vender un producto. Sin embargo, es a esta edad cuando tienen capacidad para realizar la compra independiente de los productos de consumo que les atraen, como golosinas y juguetes, sin la ayuda de sus padres y de entender el concepto del dinero como medio para obtenerlos (17-18).

Teniendo en cuenta esto, las empresas han desarrollado estrategias de marketing, destinadas a informar, persuadir, vender y satisfacer a los niños como clientes (19). Un estudio del Consejo Nacional de Televisión (CNTV) demostró que un $78 \%$ de los niños entre 8 y 13 años dispone de un promedio de $\$ 300$ (0.6 USD) por día para llevar a la escuela y lo utilizará para adquirir alguno de los productos que han visto en algún medio publicitario $(20,21)$. Esto concuerda con otros estudios que indican que un $31 \%$ de los padres declara dar dinero a sus hijos menores de 12 años y un $94 \%$ de los padres y madres señala consultar a sus hijos acerca de las decisiones de consumo que los afectan, potenciándolos como un grupo importante de consumidores $(22,23)$.

Los niños son vistos tempranamente, como un mercado atractivo debido al dinero que pueden gastar y al dinero que gastarán como adultos, ya que a futuro serán quiénes tomarán directamente las decisiones de consumo de productos y servicios. Por esto, las campañas publicitarias buscan llamar la atención de sus marcas y productos a temprana edad, estimulando la lealtad futura de estos consumidores hacia dichas marcas (24).

El objetivo general de este estudio fue describir la Publicidad Alimentaria emitida por los canales asociados a la Asociación Nacional de Televisión (ANATEL), en el horario comprendido entre las 8.00 y las 22.00 horas, durante una semana del mes de enero y una semana de febrero del año 2010. Los objetivos específicos fueron; observar, registrar e identificar la programación de los canales de televisión asociados a ANATEL; clasificar la publicidad alimentaria emitida como saludable, medianamente saludable o no saludable; y determinar la frecuencia de emisión y tiempo de duración de la publicidad alimentaria; saludable, medianamente saludable y no saludable, en el total de horas de publicidad en la programación de los canales de televisión asociados a ANATEL.

\section{MATERIAL Y MÉTODOS}

Se utilizó un diseño no experimental transeccional descriptivo. La población objetivo corresponde a la programación de lunes a domingo, de los canales de televisión asociados a ANATEL: Televisión Nacional de Chile (TVN), Canal 13, Universidad Católica de Valparaíso (UCV), La Red, Mega, Telecanal, ChileVisión (CHV). La muestra de televisión extraída se conformó de 23.520 minutos de transmisión que quedó compuesta por 3.406,1 minutos destinados a publicidad, que representan 6.425 comerciales incluidos en el estudio. De la población total seleccionada se eligió la programación emitida de lunes a domingo, entre las 8.00 y 22.00 , durante una semana de enero y una semana de febrero del año 2010, como horario estandarizado para todos los canales, abarcando 14 horas diarias de programación para cubrir los horarios de mayor programación y exposición de la población infantil a la TV. Las variables medidas en el estudio fueron, publicidad alimentaria (variable dependiente), frecuencia y tiempo de los anuncios publicitados (variable independiente) y product placement (variable interviniente no controlada). Las definiciones para las variables independientes fueron:

- Frecuencia. Definición conceptual: frecuencia $(\mathrm{F})$, medida que se utiliza para indicar el número de repeticiones de cualquier fenómeno o suceso periódico en la unidad de tiempo.

- Definición operacional; número de veces que se repite un anuncio publicitario o comercial en un determinado tiempo de programación.

- Tiempo (T) Definición conceptual; magnitud con la que se mide la duración de un determinado fenómeno o suceso, en segundos según el Sistema Internacional de Unidades (SI).

- Definición operacional; medir con un cronómetro la duración en segundos de un anuncio publicitario o comercial en la programación, transformándolo a minutos para un mejor tratamiento de la información.

Los datos se recolectaron por medio de observación estructurada con tres observadores distintos, no participantes. El registro de la publicidad se realizó durante dos semanas, con días de semana distintos para 
cada canal a grabar y un sábado y un domingo elegidos aleatoriamente. Para clasificar los comerciales se hizo una observación y registro directo de la programación y de las tandas comerciales considerando el tiempo de duración. La recolección de los datos se realizó aplicando instrumentos previamente diseñados.

Clasificación de los alimentos. Para clasificar los alimentos publicitados en alimentos saludables, medianamente saludables y no saludables se establecieron criterios en base al contenido de nutrientes críticos; grasa total, grasa saturada, hidratos de carbono simples y sodio, por cada $100 \mathrm{~g} \mathrm{o} 100 \mathrm{ml}$ tomando como referencia la tabla de Clasificación de la Agencia Nacional de Alimentos de Inglaterra, denominada "semáforo nutricional" (tabla 1). Se definieron como alimentos saludables, si poseen todos los nutrientes críticos en rango de bajo contenido; medianamente saludable, si poseen al menos un nutriente crítico en el rango mediano contenido o tiene nutrientes en bajo contenido y ninguno en alto contenido y no saludable, si poseen al menos un nutriente crítico en alto contenido.

Una vez seleccionada la muestra compuesta por la publicidad alimentaria emitida, se adquirieron los productos publicitados para analizar el etiquetado nutricional y clasificar la publicidad alimentaria de acuerdo a los criterios definidos.

\section{ANÁLISIS ESTADÍSTICO}

El tratamiento estadístico de los datos obtenidos en el estudio, se realizó por medio del programa Microsoft Office Excel 2003, considerando distribución de frecuencias y porcentajes de los datos analizados. Se basó en un análisis descriptivo, previa tabulación de datos según; duración de los comerciales, clasificación de los comerciales, y análisis del etiquetado nutricional. Se analizó la totalidad de la programación, sin distinción de canales de televisión.

\section{RESULTADOS}

Frecuencia y tiempo de programación de la publicidad. Del registro de la publicidad, considerando dos días de semana distintos para cada canal y un sábado y un domingo de las dos semanas programadas para tal efecto, se obtuvo un total de 23.520 minutos de publicidad total, de los cuales, 3.406,1 minutos correspondieron a programación publicitaria, (14\%) del total de la muestra y 20.113,9 minutos de programación no publicitaria (86\%). De los 3406,1 minutos de programación publicitaria, se registró una frecuencia de 6.425 anuncios, de estos anuncios un 16\% correspondió a publicidad alimentaria con una frecuencia de 1045 anuncios y con un tiempo de programación de 463 minutos (14\%).

Frecuencia y tiempo de la emisión de la publicidad alimentaria. La publicidad alimentaria registrada se clasificó en base a los criterios señalados previamente, en publicidad alimentaria saludable, medianamente saludable y no saludable, diferenciada por día de semana y fin de la semana. El análisis de la publicidad alimentaria, mostró que la publicidad alimentaria saludable representó a un $8 \%$ (38,5 minutos) y un total de 89 anuncios (9\%). La publicidad medianamente saludable representó un $30 \%$ de la publicidad alimentaria emitida, con un total de 284 anuncios $(27 \%)$ y un tiempo de registro de 138,9 minutos y un $62 \%$ de la publicidad correspondió a

\section{TABLA 1}

\section{Criterios nutricionales para clasificar alimentos saludable, medianamente saludable y no saludable según contenido de nutrientes críticos.}

\section{NUTRIENTES}

GRASA

GRASAS SATURADAS

H. DE C
(azúcares simples agregados)

SODIO

\section{BAJO CONTENIDO}

$\leq 1,5 \mathrm{~g} / 100 \mathrm{~g}$

$$
\leq 1,5 \mathrm{~g} / 100 \mathrm{ml}
$$

$$
\leq 0,75 \mathrm{~g} / 100 \mathrm{~g}
$$
$\leq 0,75 \mathrm{~g} / 100 \mathrm{ml}$

$$
\begin{aligned}
& \leq 2,5 \mathrm{~g} / 100 \mathrm{~g} \\
& \leq 2,5 \mathrm{~g} / 100 \mathrm{ml}
\end{aligned}
$$

$$
\begin{aligned}
& \leq 0,3 \mathrm{~g} / 100 \mathrm{~g} \\
& \leq 0,3 \mathrm{~g} / 100 \mathrm{ml}
\end{aligned}
$$

\section{MEDIANO CONTENIDO}

$$
\begin{aligned}
& \geq 1,5 \mathrm{y} \leq 10 \mathrm{~g} / 100 \mathrm{~g} \\
& \geq 1,5 \mathrm{y} \leq 10 \mathrm{~g} / 100 \mathrm{ml} \\
& \geq 0,75 \mathrm{y} \leq 2,5 \mathrm{~g} / 100 \mathrm{~g} \\
& \geq 0,75 \mathrm{y} \leq 2,5 \mathrm{~g} / 100 \mathrm{ml}
\end{aligned}
$$

$$
\geq 2,5 \mathrm{y} \leq 10 \mathrm{~g} / 100 \mathrm{~g}
$$$$
\geq 2,5 \mathrm{y} \leq 7,5 \mathrm{~g} / 100 \mathrm{ml}
$$

$$
\geq 0,3 \text { y } \leq 0,5 \mathrm{~g} / 100 \mathrm{~g}
$$$$
\geq 0,3 \mathrm{y} \leq 0,5 \mathrm{~g} / 100 \mathrm{ml}
$$

\section{ALTO CONTENIDO}

$$
\geq 10 \mathrm{~g} / 100 \mathrm{~g}
$$$$
\geq 10 \mathrm{~g} / 100 \mathrm{ml}
$$

$\geq 2,5 \mathrm{~g} / 100 \mathrm{~g}$ $\geq 2,5 \mathrm{~g} / 100 \mathrm{ml}$ $\geq 10 \mathrm{~g} / 100 \mathrm{~g}$ $\geq 7,5 \mathrm{~g} / 100 \mathrm{ml}$ $\geq 0,5 \mathrm{~g} / 100 \mathrm{~g}$ $\geq 0,5 \mathrm{~g} / 100 \mathrm{ml}$

Fuente: Agencia de Normas de Alimentos (Food Stand Agency) del Reino Unido. 
publicidad alimentaria no saludable con un total de 672 anuncios (64\%) y con un tiempo de registro de 285,15 minutos (figura 1 y 2 ).
Frecuencia y tiempo de programación según tipo de publicidad y día de la semana. En relación al total de la publicidad emitida, se observó que la frecuencia de

\section{FIGURA 1}

Tiempo de duración de la publicidad alimentaria según clasificación saludable, medianamente saludable y no saludable, expresado en minutos.

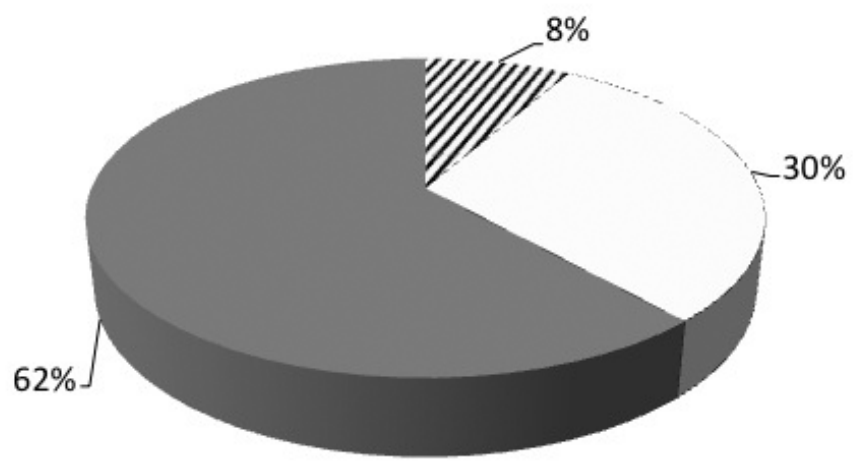

\% Publicidad Alimentaria Saludable

$\square$ Publicidad Alimentaria Medianamente Saludables

- Publicidad Alimentaria No Saludable

\section{FIGURA 2}

Frecuencia de anuncios emitidos de la publicidad alimentaria según clasificación saludable, medianamente saludable y no saludable.

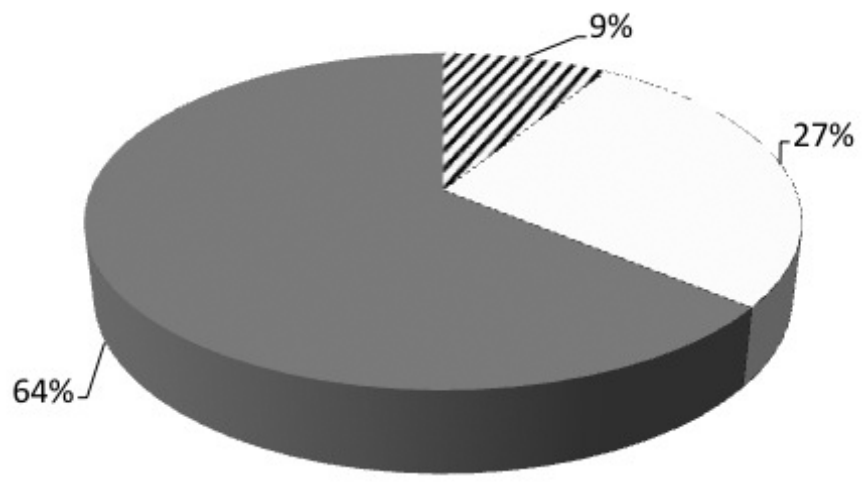

\% Publicidad Alimentaria Saludable

$\square$ Publicidad Alimentaria Medianamente Saludable

- Publicidad Alimentaria No Saludable 
la publicidad alimentaria es levemente mayor en los días de semana respecto al fin de semana con una frecuencia de 527 y 518 anuncios, respectivamente. El análisis del tipo de publicidad alimentaria muestra que la duración de la publicidad alimentaria saludable es levemente mayor en los días de semana con una duración de 22,1

\section{FIGURA 3}

Tipo de publicidad emitida por los canales de televisión asociados a ANATEL, diferenciado por día de semana y fin de semana. Medido según frecuencia $\left(\mathrm{n}^{\circ}\right)$.

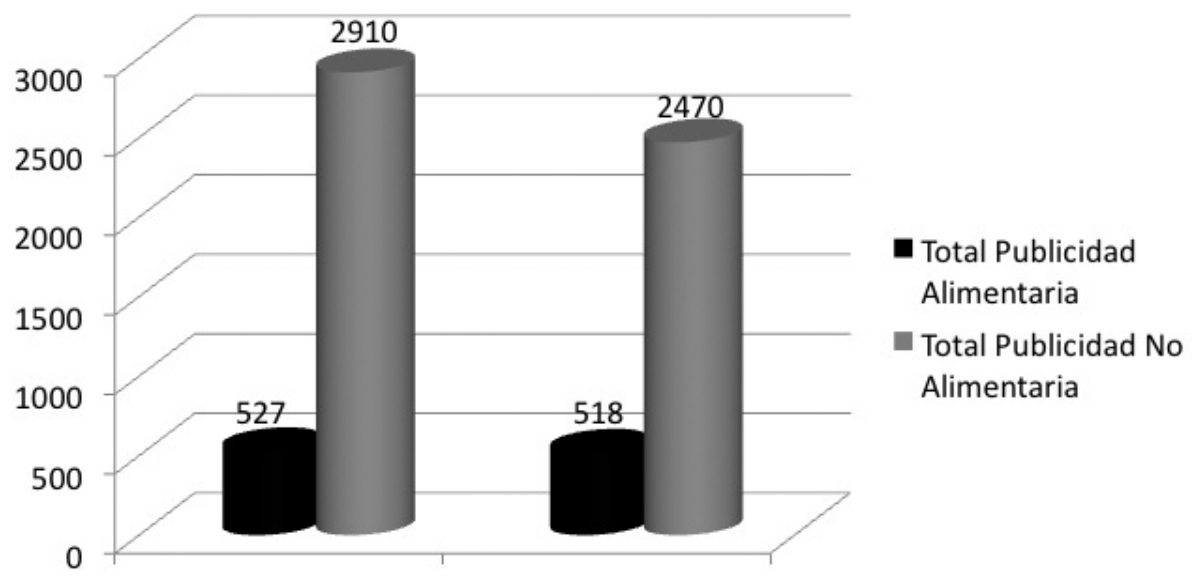

Semana

Fin de Semana

\section{FIGURA 4}

Duración de la publicidad alimentaria emitida por los canales de televisión asociados a ANATEL, diferenciado por día de semana y fin de semana, expresado en minutos.

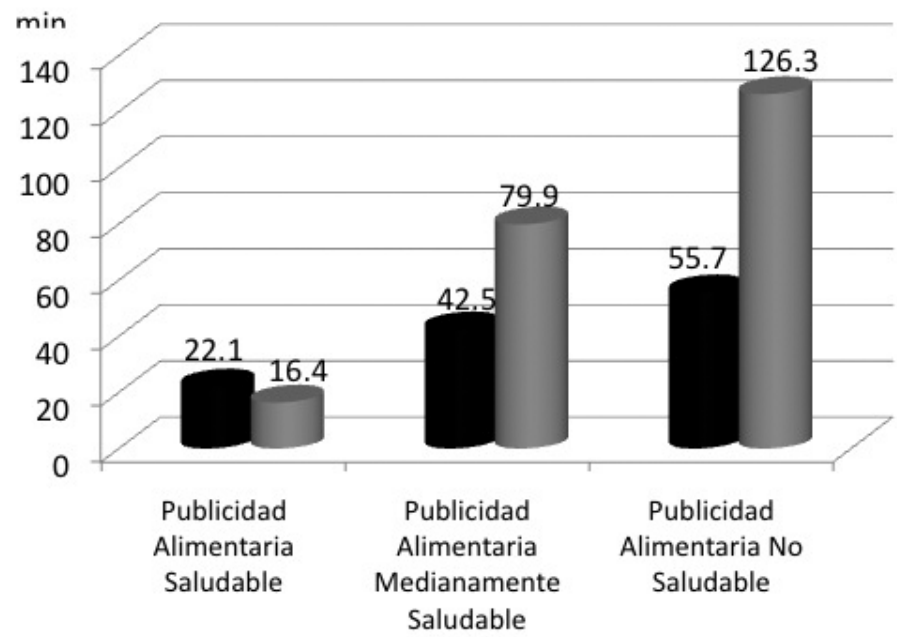

- Semana

- Fin de Semana 
minutos en día de semana versus 16,4 minutos los días de fin de semana; la publicidad alimentaria medianamente saludable es mayor los días de fin de semana, con un aumento de un $46,8 \%$ y un $127 \%$ respectivamente, respecto al total de la publicidad no alimentaria (figuras 3 y 4). A modo de resumen se presenta la tabla 2.

Tipo de productos alimentarios publicitados. El registro de los productos alimentarios publicitados permite conocer el tipo de productos publicitados en los canales asociados a ANATEL y la frecuencia de su emisión. Se observa que el mayor porcentaje correspondió a bebidas gaseosas (20\%), seguidos de los lácteos (13\%), aguas minerales $(11 \%)$ y productos tipo bocadillo $(9 \%)$, lo que en conjunto, equivale al $53 \%$ de la publicidad alimentaria emitida (figura 5).

\section{DISCUSIÓN Y CONCLUSIONES}

La prevalencia e incidencia de obesidad y sobrepeso ha aumentado de manera progresiva, tanto en países desarrollados como en vías de desarrollo, donde en los últimos 20 años las tasas de obesidad se han triplicado, situación que afecta transversalmente a todos los grupos etáreos, por género y condición socioeconómica. En América Latina y en Chile, la realidad no difiere de la situación mundial, encontrándose en la etapa de posttransición nutricional caracterizada por el predominio de sobrepeso y obesidad, con un patrón dietario con mayor cantidad de grasas saturadas, azúcares simples, alimentos procesados, consumo insuficiente de frutas, verduras, legumbres y cereales integrales $(25,26)$. Distintos estudios señalan que un cambio en los hábitos alimentarios y en la actividad física pueden influir en varios de los factores de riesgo para la población en general $(27,28)$.

Por esto, la Organización Mundial de la Salud (OMS), ha desarrollado la Estrategia Global sobre Régimen Alimentario, Actividad Física y Salud (DPAS) aprobada en la $57^{\mathrm{a}}$ Asamblea Mundial de la Salud celebrada en 2004 (8). El principal objetivo de la DPAS radica en fomentar y proteger la salud con el fin de reducir las ECNT, limitando el consumo de nutrientes críticos de la población que padece de sobrepeso u obesidad (grasas saturadas y ácidos grasos trans, sodio, azúcares y fibra). También se orienta hacia el análisis del etiquetado de los alimentos, sus propiedades saludables y el marketing, ya que estos nutrientes críticos destacan dentro de los alimentos que se publicitan en medios de comunicación masiva y que afectan la toma de decisión al momento de la elección de los alimentos y afecta la actividad física.

Diversos estudios indican que el número de horas diarias destinadas por niños y niñas a ver televisión tiene asociación directa con la falta de actividad física, menor gasto energético basal e inadecuada ingesta alimentaria y a una mayor exposición a publicidad alimentaria que promueve consumos de alimentos no saludables, cuya frecuencia es mayor respecto a alimentos saludables. La mayor exposición a una publicidad induce a la elección

\section{TABLA 2}

\section{Clasificación, tiempo de duración y frecuencia de anuncios de publicidad alimentaria emitida por los canales de televisión asociados a Anatel, diferenciado por día de semana y fin de semana.}

\section{TOTAL \\ SEMANA \\ FIN DE SEMANA}

\begin{tabular}{|c|c|c|c|c|c|c|c|c|c|c|c|c|}
\hline & \multicolumn{2}{|c|}{ Frecuencia } & \multicolumn{2}{|c|}{ Tiempo } & \multicolumn{2}{|c|}{ Frecuencia } & \multicolumn{2}{|c|}{ Tiempo } & \multicolumn{2}{|c|}{ Frecuencia } & \multicolumn{2}{|c|}{ Tiempo (min) } \\
\hline & $\mathbf{n}^{0}$ & $\%$ & $\min$ & $\%$ & $\mathbf{n}^{\mathbf{o}}$ & $\%$ & $\min$ & $\%$ & $\mathbf{n}^{0}$ & $\%$ & $\min$ & $\%$ \\
\hline $\begin{array}{l}\text { Publicidad } \\
\text { alimentaria saludable }\end{array}$ & 89 & 9 & 38,5 & 8 & 53 & 59,6 & 22,1 & 57,4 & 36 & 40,4 & 16,4 & 42,6 \\
\hline $\begin{array}{l}\text { Publicidad alimentaria } \\
\text { medianamente saludable }\end{array}$ & 284 & 27 & 138,9 & 30 & 133 & 46,8 & 42,5 & 79,9 & 151 & 53,2 & 79,9 & 57,5 \\
\hline $\begin{array}{l}\text { Publicidad alimentaria } \\
\text { no saludable }\end{array}$ & 672 & 64 & 285,2 & 62 & 341 & 50,7 & 55,7 & 126,3 & 331 & 49,3 & 126,3 & 44,3 \\
\hline $\begin{array}{l}\text { Total publicidad } \\
\text { alimentaria }\end{array}$ & 1045 & 100 & 463 & 100 & 527 & 50 & 52 & 223 & 518 & 50 & 223 & 48 \\
\hline $\begin{array}{l}\text { Total publicidad } \\
\text { no alimentaria }\end{array}$ & 5380 & 100 & 2943 & 100 & 2910 & 54 & 1641 & 56 & 2470 & 46 & 1302 & 44 \\
\hline
\end{tabular}


de los productos publicitados $(17,19,21,23)$.

Dada esta situación se han elaborado códigos internacionales de regulación de la publicidad alimentaria. Países como Suecia, Noruega, Canadá y España poseen una legislación que prohíbe la publicidad de productos potencialmente dañinos para niños y niñas menores de 12 años (29). En Chile, en el año 1986, la Asociación Chilena de Agencias de Publicidad (ACHAP) y la Asociación Nacional de Avisadores (ANDA), aprobaron el texto original del Código Chileno de Ética Publicitaria, que recoge los principios y normas éticas que deben imperar en la práctica publicitaria.

Posteriormente, en 1987, se constituyó el Consejo Nacional de Autorregulación Publicitaria (CONAR), organismo rector en la materia y, a partir del 2009 se tramita la aprobación del proyecto de ley sobre composición nutricional de los alimentos y su publicidad, que con fecha 19/07/2011 fue aprobado el veto presidencial por votación unánime en la Comisión de Salud del Senado, referente a la aplicación tanto para alimentos envasados y preparados de un rótulo especial para resaltar el contenido de nutrientes críticos, la venta de alimentos en instituciones educacionales básicas y medias de alimentos determinados como perjudiciales para la salud, la inclusión de educación nutricional y 3 bloques semanales de educación física a nivel escolar.
Respecto a la publicidad, se señala que se regulará como medio de comunicación sólo a la televisión y se eliminarán los ganchos comerciales para atraer la compra de los productos. (30 - 33).

Respecto al etiquetado de los alimentos y la declaración del contenido nutricional de los alimentos que se comercializan envasados, el decreto supremo $\mathrm{N}^{\circ} 57$ del 16.02.05, indica la modificación del Reglamento Sanitario de los Alimentos, incorporando la obligatoriedad de incluir este rotulo llamado, "etiquetado nutricional. Es así como los consumidores tienen el derecho de conocer claramente el aporte nutricional de los alimentos que adquirirán, la industria alimentaria tiene el deber de declarar en el etiquetado nutricional el aporte de energía, contenido de proteínas, grasas o lípidos, hidratos de carbono, vitaminas, minerales o factores alimentarios, fibra dietética y colesterol $(33,34)$.

Algunas de las recomendaciones prácticas para el consumidor están especificadas en los descriptores nutricionales, según el aporte de energía, nutriente o factor alimentarios declarado en el etiquetado. Estos descriptores buscar guiar al consumidor en la elección de los alimentos informando sobre sus propiedades nutricionales, sin embargo no declaran que el alimento sea saludable o no.

Esta situación, impulsó a que en agosto del año 2010

\section{FIGURA 5}

Tipo de alimentos publicitado en el total de publicidad alimentaria emitida por los canales asociados a ANATEL, medido en frecuencia.

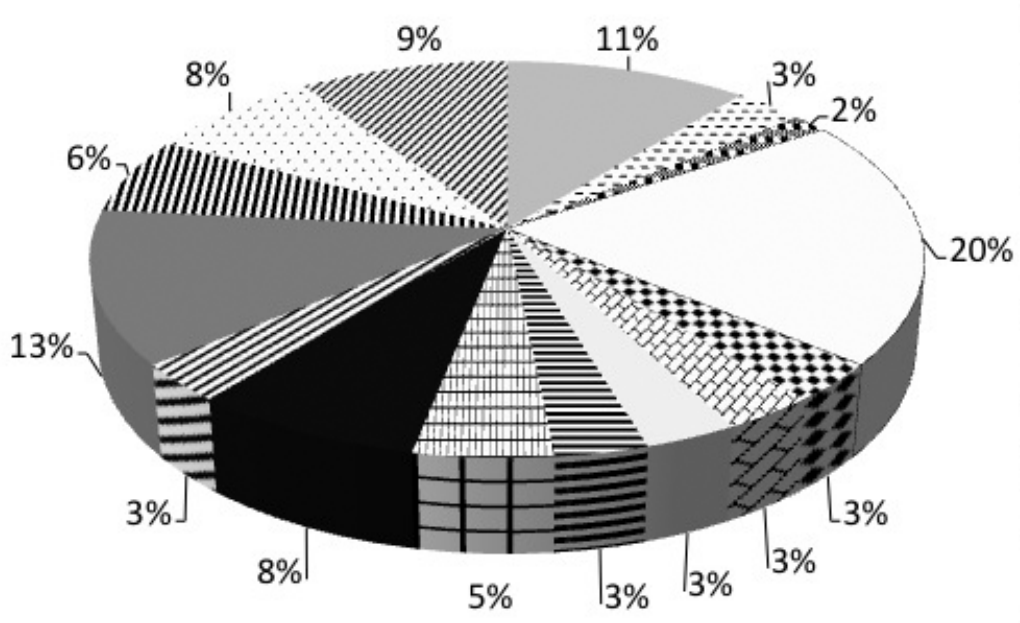

Aguas minerales

- Bebidas calientes

Bebidas frías

$\square$ Bebidas gaseosas

- Cereales para el desayuno

$\checkmark$ Chocolates

Galletas

$=$ Aceites y grasas

1 Helados

- Jugos en polvo

Jugos néctar

- Lácteos

11 Postres instantáneos

Productos cárneos

$>$ Productos snack 
se modificara el decreto 977 de 1996 del Reglamento Sanitario de los Alimentos con la finalidad de esclarecer la información que se declara en el etiquetado nutricional de los alimentos (35). En este contexto, la nueva ley aprobada en la Comisión de Salud del Senado en junio de este año, establece en primer término un protocolo de acuerdo para enviar un proyecto de ley que regule la publicidad de los alimentos que resulten definidos por el Ministerio de Salud como "altos en sal", "altos en azúcares" o "altos en grasa" en el sentido que su difusión en televisión no podrá efectuarse antes de las 10 de la noche, con la excepción de los eventos de carácter deportivo, cultural o artístico y que sea imposible fiscalizar, lo que constituye un avance en la información y protección del consumidor (36).

Los resultados de esta investigación muestran la realidad de la publicidad alimentaria en Chile, donde de un total de 23.520 minutos de programación observada, un $14 \%$ es programación publicitaria, de ésta sólo el $16 \%$ corresponde a publicidad alimentaria. A partir de la clasificación de los productos alimentarios publicitados en base a los criterios definidos, se aprecia que la mayoría de los productos publicitados son no saludables, los cuales presentan altos niveles de nutrientes críticos (grasas totales, grasa saturada, sodio y azucares simple).

De la publicidad alimentaria que consume la población chilena, se observó que ésta dedica mayor porcentaje de tiempo a publicidad no saludable, en segundo lugar a publicidad medianamente saludable y en menor porcentaje de frecuencia de anuncios y tiempo de emisión a publicidad alimentaria saludable. Se observó que no existe diferencia significativa en los días de emisión de la publicidad alimentaria, siendo levemente mayor en días de semana con respecto al fin de semana. Destaca que en Chile no existen criterios de clasificación para determinar si un alimento es saludable, medianamente saludable y no saludable, de acuerdo al contenido de nutrientes críticos.

Las familias chilenas comparten gran parte de su tiempo frente al televisor, la publicidad emitida por los canales de televisión por la frecuencia y tiempo de duración incentiva el consumo de los alimentos más publicitados que corresponden en su mayoría a los de mayor riesgo nutricional por el contenido de nutrientes críticos perjudiciales para la salud. La exposición constante a este tipo de publicidad impacta en la conducta de la población, la que se transforma en hábitos de vida poco saludables en desmedro de su salud física, psicológica y social.

Es de esperar, que la nueva ley de etiquetado y publicidad de alimentos, se apruebe en un corto tiempo y resguarde de la exposición a la publicidad alimentaria no saludable, en especial a los grupos de menor edad por la vulnerabilidad que presentan en la etapa de desarrollo y crecimiento, en que se requiere que reforcemos conductas y hábitos saludables que perduren en el tiempo y contribuyamos a generar ciudadanos con una salud compatible con una mejor calidad de vida.

\section{RESUMEN}

El objetivo de este estudio, fue describir la Publicidad Alimentaria emitida por los canales pertenecientes a la Asociación Nacional de Televisión. En una semana de enero y una de febrero del año 2010 se seleccionó y clasificó la publicidad alimentaria emitida como saludable, medianamente saludable y no saludable, en base al contenido de nutrientes críticos; grasa total, grasa saturada, hidratos de carbono simples y sodio. Se utilizó como criterio de referencia, la tabla de clasificación de la Agencia Nacional de Alimentos de Inglaterra, denominada "semáforo nutricional". Se registraron y analizaron 6425 anuncios correspondientes a 56 horas 36 minutos continuas de tiempo, de ellos, se identificaron 1045 anuncios alimentarios (16\%), equivalente en tiempo a 7 horas 42 minutos (14\%) del total. Se examinaron las etiquetas nutricionales de los productos para la selección y calificación en saludable, medianamente saludable y no saludable. Se observó, que del total de la publicidad alimentaria, el $64 \%$ correspondió a publicidad alimentaria no saludable; el $27 \%$, a publicidad alimentaria medianamente saludable y, el $9 \%$ publicidad alimentaria saludable, con un tiempo de emisión de un $62 \%, 30 \%$ y $8 \%$, respectivamente. La publicidad alimentaria emitida es principalmente no saludable, promueve el consumo de alimentos altos en grasa, azúcar y sodio. La televisión al emitir esta publicidad promueve e incentiva a la población a llevar estilos de vida no saludables, en desmedro de la salud de la población

Palabras clave: publicidad alimentaria, etiquetado nutricional, grasa, azúcar, sodio.

Dirigir la correspondencia a:

Profesora

Mirta Crovetto Mattassi

Facultad de Ciencias de la Salud.

Universidad de Playa Ancha

Avenida Playa Ancha 850

Valparaíso

Chile

Fax: ( 56-32) 2286713

Email: mcrovetto@upla.cl

\section{BIBLIOGRAFÍA}

1. Vio F, Albala C, Kain J. Nutrition transition in Chile 
revisited: mid-term evaluation of obesity goals for the period 2000-2010.Public Health Nutr 2008; 11(4):405-12.

2. Ministerio de Salud. Informe final: Estudio de carga de enfermedad y carga atribuible. Santiago, Chile: MINSAL, 2008.

3. Luque C, Cisternas F, Araya M. Cambios del patrón de enfermedad en la postransición epidemiológica en salud en Chile, 1950-2003. Rev Méd Chil. 2006; 134:703-12.

4. Cruchet S., Rozowski J. Obesidad un enfoque integral. Nestlé Chile, Santiago, 2007.

5. Braguinsky J. Prevalencia de obesidad en América Latina. An. Sist. Sanit. Navar. 2002; 25 Suppl 1:109-15.

6. Ministerio de Educación, Junta Nacional de Auxilio Escolar y Becas. Estado nutricional de escolares chilenos de primer añohásico Santiago Disponible en Internet: http://www.junaeb.cl/mapa nutriciohal. 2009.

7. Domínguez-Vásquez P, Olivares S, Santos JL. Influencia familiar sobre la conducta alimentaria y su relación con la obesidad infantil. Arch Latinoam Nutr 2008; 58(3):249-55.

8. Organización Mundial de la Salud. Estrategia mundial sobre régimen alimentario, actividad física y salud. 57 Asamblea Mundial de la Salud. Resolución AMS WHA57.17. Ginebra, 2004.

9. McNeal, J. Los niños como consumidores de productos sociales y comerciales. Organización Panamericana de la Salud (OPS), Washington D.C, USA. 2000.

10. Olivares S, Albala C, Garcia F, Jofre I. Publicidad televisiva y preferencias alimentarias en escolares de la Región Metropolitana. Rev Med Chil 1999; 127:791-9.

11. Consejo Nacional de Televisión (CNTV) Estudio de Audiencia y Consumo Televisivo 1999-2002. Santiago, 2003.

12. Escavias De Carvajal M. Piden más control de la publicidad alimentaria para niños y jóvenes. Rev Correo Farmacéutico. Sección Medicina. 2009; 18:27.

13. McDermott L, Stead M, Hastings G. A marketing strategy to review the effects of food promotion to children. In: G Hastings. Social Marketing. Why should the devil have all the best tunes? London, UK, Elsevier 2007.

14. Bustos N, Kain J, Leyton B, Olivares S, Vio F. Colaciones habitualmente consumidas por niños de escuelas municipalizadas: Motivaciones para su elección. Rev Chil Nutr 2010; 37(2):178-83.
15. Consejo Nacional de Televisión (CNTV). Estudio Estadístico de Televisión Abierta 2002-2004 San-

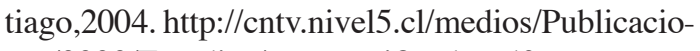
nes/2003/Estudios/tweenunificado.pdf

16. Mpore, E. S., W.L. Wilkie and R.J. Lutz. Passing the Torch: Intergenerational Influences as a Source of Brand Equity. Journal of Marketing 2002; 66:17-37.

17. Olivares S, Bustos N, Moreno X, Lera L, Cortez S. Actitudes y prácticas sobre alimentación y actividad física en niños obesos y sus madres en Santiago, Chile. Rev Chil Nutr 2006; 33:170-9.

18. Consumers International. Campañas "Generación de Comidas Chatarras. ¿Qué es la obesidad? Estadísticas" Consumers International, London, UK 2011 htp / / es consumersinternationalorg/media/573539 generacioncomidachatarraconjuntodeherramientas. pdf

19. Фonsumers International. Con gusto a poco: Políticas de las empresas alimentarias sobre la promoción dirigida a los niños. Consumers International London, UK, 2009.

20. Consejo Nacional de Televisión (CNTV). "Zoom Tween:: 3 Estudios Cualitativos" Departamento de Estudios Santiago, 2004 htp $/ /$ enty nivel $5 \mathrm{cl}$ medios/Publicaciones/2003/Estudios/tweenunificado.pdf

21. Uribe R. Características de la publicidad infantil chilena en tiempos de mayor acceso a los medios y a los bienes de consumo. Universidad de Chile. Estudios de Administración 2005;12:2.

22. Kain J, Uauy R, Leyton B, Cerda R, Olivares S, Vio F. Efectividad de una intervención en educación alimentaria y actividad física para prevenir obesidad en escolares de la ciudad de Casablanca, Chile (2003-2004). Rev Med Chil 2008; 136:22-30.

23. Mehta K. Obesidad infantil: el precio inaceptable del éxito publicitario. Diabetes Voice 2007; 52:38-9.

24. Jarlbro, G., "Children and Television Advertising: The players, the Arguments and the Research during the Period 1994-2000 Konswmentverket Stackholm, Sweden 2001 ht p.//www nordicom. gu.se/common/publ_pdf/34_Jarlbro.pdf

25. Vio F, Albala C. Nutrition transition in Chile: a case study. In: Globalization of food systems in developing countries: impact on food security and nutrition. Food and Agriculture Organization of the United Nations, Rome. FAO Food Nutr Paper 2004; 83:275-84.

26. Organización Mundial de la Salud. Organización para la Alimentación y Agricultura. Dieta, nutrición y prevención de enfermedades crónicas. Informe de 
una Consulta Mixta de Expertos. Serie de Informes Técnicos OMS/FAO 916. Ginebra, 2003.

27. Crovetto M, Uauy R. Cambios en la disponibilidad de alimentos en el Gran Santiago por quintiles de ingreso.1988-1997. Arch Latinoam Nutr 2008; 58:40-8.

28. Crovetto M. Cambios en la estructura alimentaria y consumo aparente de nutrientes de los hogares del Gran Santiago 1988-1997. Rev Chil Nutr 2002; 29:24-32.

29. Agencia Española de Seguridad Alimentaria y Nutrición. Entra en vigor el Código PAOS de autorregulación de la publicidad destinada a menores para combatir la obesidad infantil Noviembre 2005 Disponible en Internet. hitp.//wwwaesan mse es/ AESAN/web/notas_prensa/vigor_PAOS.shtml

30. Consejo de Autorregulación y Etica Publicitaria. Código de Ética_Publicitaria_Directorio CONAR,

31. Consejo Nacional de Televisión, Mc Cannan Erickson, Junta Nacional de Jardines Infantiles. Informe Preescolares 0-5 - Tons Chilenos $2007 \mathrm{htth} / 1$ www cntvel/prontus_entv/site/artic/20110323/ asocfile/20110323160701/librotoons2007.pdf
32. Consejo de Autorregulación y Etica Publicitaria. La Autorregulación_de la_Publicidad_en Chile Santiago, 2010 ht H.//www conarcl/2010/12/lahytorregulacion-de-la-publicidad-en-chile/

33. Girardi G. Proyecto de ley sobre regulación de alimentos pasaludables 2009 Disponjble en: http:// sil.congreso.cl/docsil/proy5308.doc. Accedido 29 de Julio de 2011.

34. Zacarías I. y Vera G. Selección de Alimentos: Uso del Etiquetado Nutricional para una Alimentación Saludable. Manual de consulta para profesionales de la salud. MINSAL, Santiago, 2005 Disponible en Internet · hep//www nutrinfo com/pagina/info/ manual_etiquetado_nutricional.pdf

35. Diario Oficial de la República de Chile. Ministerio de Salud, Subsecretaría de salud Pública. Decreto No88.- Modifica decreto No 977, de 1996, Reglamento Sanitario de los Alimentos. Santiago 2011, p.4.

36. Senado de Chile. Ley sobre composición nutricional de los alimentos y su publicidad. Informe Comisión de Salud, Boletín No 4921-11 hrtp://sil.senado. cl/cgi-bin/index_eleg.pl?4921-11. Disponible en internet. Accedido 2 de agosto de 2011. 